\title{
Regorafenib as a potential adjuvant chemotherapy agent in disseminated small colon cancer: Drug selection outcome of a novel screening system using nanoimprinting 3-dimensional culture with HCT116-RFP cells
}

\author{
YUKIE YOSHII $^{1}$, TAKAKO FURUKAWA ${ }^{1}$, HIRONORI AOYAMA $^{1,2}$, NAOYA ADACHI $^{1,2}$, MING-RONG ZHANG $^{1}$, \\ HIDEKATSU WAKIZAKA ${ }^{1}$, YASUHISA FUJIBAYASHI ${ }^{1}$ and TSUNEO SAGA $^{1}$ \\ ${ }^{1}$ Molecular Imaging Center, National Institute of Radiological Sciences, Chiba 263-8555; \\ ${ }^{2}$ Faculty of Science, Toho University, Funabashi 274-8510, Japan
}

Received November 13, 2015; Accepted December 18, 2015

DOI: $10.3892 /$ ijo.2016.3361

\begin{abstract}
Colon cancer is one of the leading causes of cancer death worldwide. Adjuvant chemotherapy following primary surgical treatment is suggested to be beneficial in eradicating invisible disseminated small tumors in colon cancer; however, an effective drug remains to be developed. Recently, we reported a novel drug screening system using a nanoimprinting 3-dimensional (3D) culture that creates multicellular spheroids, which simulate in vivo conditions and, thereby, predict effective drugs in vivo. This study aimed to perform drug selection using our recently developed 3D culture system in a human colon cancer HCT116 cell line stably expressing red fluorescent protein (HCT116-RFP), to determine the most effective agent in a selection of clinically used antitumor agents for colon cancer. In addition, we confirmed the efficacy of the selected drug regorafenib, in vivo using a mouse model of disseminated small tumors. HCT116-RFP cells were cultured using a nanoimprinting 3D culture and in vitro drug selection was performed with 8 clinically used drugs [bevacizumab, capecitabine, cetuximab, 5-fluorouracil (5-FU), irinotecan, oxaliplatin, panitumumab and regorafenib]. An in vivo study was performed in mice bearing
\end{abstract}

Correspondence to: Dr Yukie Yoshii, Molecular Imaging Center, National Institute of Radiological Sciences, Anagawa 4-9-1, Inage, Chiba 263-8555, Japan

E-mail: yukiey@nirs.go.jp

Abbreviations: ANOVA, analysis of variance; FLT, 3'-[ $\left[{ }^{18} \mathrm{~F}\right]$ fluoro-3'deoxythymidine; $\mathrm{IC}_{50}$, half-maximal inhibitory concentration; NCP, nanoculture plate; PET, positron emission tomography; RFP, red fluorescent protein; SD, standard deviation; SUV, standard uptake value; 3D, 3-dimensional; 5-FU, 5-fluorouracil

Key words: colon cancer, drug selection, nanoimprinting 3-dimensional culture, regorafenib, disseminated small tumors
HCT116-RFP intraperitoneally disseminated small tumors using 3 '- $\left[{ }^{18} \mathrm{~F}\right]$-fluoro-3'-deoxy thymidine-positron emission tomography and fluorescence microscopy imaging to evaluate the therapeutic effects. Regorafenib was determined to be the most effective drug in the 3D culture, and significantly inhibited tumor growth in vivo, compared to the untreated control and 5-FU-treated group. The drug 5-FU is commonly used in colon cancer treatment and was used as a reference. Our results demonstrate that regorafenib is a potentially efficacious adjuvant chemotherapeutic agent for the treatment of disseminated small colon cancer and, therefore, warrants further preclinical and clinical studies.

\section{Introduction}

Colon cancer, also known as colorectal cancer, is one of the leading causes of cancer deaths in men and women worldwide (1). In colon cancer, adjuvant chemotherapy after the primary surgical intervention is suggested to be beneficial in eradicating the visually undetectable disseminated small tumors and improving the prognosis (2). The nucleic acid antimetabolite 5-fluorouracil (5-FU) has mainly been used for adjuvant chemotherapy in colon cancer treatment. However, adjuvant chemotherapeutic clinical studies using 5-FU alone or in combination with leucovorin or other drugs have shown limited effectiveness $(3,4)$. For example, a reduced risk of recurrence was reported, but not of death in patients with stage II cancer, and it was not effective in patients with stage III cancers $(3,4)$. Therefore, new efficacious drugs are required to improve the outcome of adjuvant chemotherapy in colon cancer treatment.

Recently, we developed a novel drug screening system using a nanoimprinting 3-dimensional (3D) culture that creates multicellular spheroids mimicking in vivo conditions $(5,6)$ (Fig. 1A). This 3D culture uses a microplate that possesses well-regulated inorganic nanoscale indented patterns printed on the base using nanoimprinting technology (nanoculture plate, NCP). The nanoimprinted pattern acts as a scaffold to which cultured cells adhered, albeit with less physical contact with the culture plates than with traditional $2 \mathrm{D}$ plates. NCPs 
were shown to facilitate spontaneous tumor cell migration, intercellular adhesion, and formation of 3D multicellular spheroids similar to in vivo tumors while maintaining cellular proliferation and viability (5). In addition, we revealed that the high-throughput drug screening using the nanoimprinting 3D culture efficiently identified effective drugs for the treatment of xenograft tumors subcutaneously transplanted in vivo, compared to $2 \mathrm{D}$ culture (6). Here, we noted that the nanoimprinting 3D culture forms adherent and active spheroids, which have a considerable resemblance to the disease condition of disseminated small tumors in vivo and subcutaneous xenografts. Therefore, in this study, we performed drug selection using the nanoimprinting 3D culture to identify an effective drug against disseminated small tumors, using a human colon cancer model of HCT116-red fluorescent protein (RFP) cells.

\section{Materials and methods}

Cell cultivation. Human colon carcinoma cell line HCT116 (CCL-247, American Type Cell Collection, ATCC) were used in this study. HCT116 cells were stably transfected with RFP lentivirus (Lenti-Red, LG502, Biogenova, Rockville, MD, USA) following the manufacturer's protocol. Then, a clone that strongly expressed the RFP was established by limiting dilution and denoted as HCT116-RFP cells. The cells were incubated in a humidified atmosphere of $5 \% \mathrm{CO}_{2}$ at $37^{\circ} \mathrm{C}$ in Dulbecco's modified Eagle's medium (DMEM, 11995-065, Invitrogen, Carlsbad, CA, USA), supplemented with $10 \%$ fetal bovine serum (FBS), and antibiotics as culture medium. Exponentially growing cells were used in the study, and were detached from the plates with trypsin. The number of viable cells was determined using the trypan blue dye exclusion method.

In vitro drug selection with NCPs. We used NCPs (96-wells, SCIVAX Life Sciences, Kawasaki, Japan) with a nanoscale square grid pattern consisting of 500-nm line width as well as 1 and $2 \mu \mathrm{m}$ line depth and spacing, respectively, printed on the transparent synthetic resinous bases, using the previously reported nanoimprinting technique (5).HCT116-RFP cells were seeded in the 96 -well NCPs at a density of $1 \times 10^{4}$ cells $/ 100 \mu \mathrm{l}$ of medium and incubated in a humidified atmosphere of $5 \% \mathrm{CO}_{2}$ at $37^{\circ} \mathrm{C}$ for 7 days to construct the spheroids. For in vitro drug selection, we used 8 antitumor agents that are clinically used for the treatment of patients with colon cancer including bevacizumab, capecitabine, cetuximab, 5-FU, irinotecan, oxaliplatin, panitumumab, and regorafenib (details shown in Table I). In the experiments, drugs were added to each well of the NCPs at a final concentration of $10,5,3,1,0.5$ and $0 \mu \mathrm{M}$ in the medium and the doses were chosen based on previous studies (6-16). The spheroids were incubated for 3 days $(n=6)$ in a $\mathrm{CO}_{2}$ incubator. During the incubation, the formation of the spheroid morphology was monitored using time-lapse analysis by acquiring images every $2 \mathrm{~h}$ at x10 magnification using an IncuCyte imaging system (Essen Bioscience, Ann Arbor, MI, USA). After a 3-day incubation, the cell viability (percentage of control cells treated with $0 \mu \mathrm{M}$ drug) was evaluated by quantifying the levels of cellular adenosine-5'-triphosphate (ATP) using the CellTiter-Glo Luminescent Cell Viability assay (Promega, Madison, WI, USA) (5). The luminescence signals were detected using a microplate reader (SpectraMax M5, Molecular Devices, Sunnyvale, CA, USA).

Ethics statement. Animal experiments in this study were carried out in strict accordance with the recommendations of the Guide for the Care and Use of Laboratory Animals of the National Institute of Radiological Sciences, Japan. The protocol was approved by the Animal Ethics Committee of the National Institute of Radiological Sciences, Japan (permit no. M40-01). All efforts were made to minimize suffering of the animals in the experiments.

In vivo antitumor study. We examined the in vivo effects of regorafenib, which was selected in the nanoimprinting $3 \mathrm{D}$ culture, using a mouse model of HCT116-RFP disseminated small tumors. The model was established in BALB/c female nude mice (6-week-old with uniform body weight) obtained from the Japan SLC (Shidzuoka, Japan). A purified diet (AIN-93M, TestDiet, St. Louis, MO, USA) was used during the experiments to avoid the effect of diet on abdominal autofluorescence detected by in vivo fluorescence microscopic imaging. Before the experiments, the mice were acclimatized for $>1$ week, and then intraperitoneally (i.p.) injected with $3 \times 10^{6}$ HCT116-RFP cells suspended in $500 \mu$ l phosphate-buffered saline (PBS). On day 5 after inoculation (day 0 of treatment), mice were randomly divided into 3 groups of 5 animals each including i) control, ii) regorafenib, and iii) 5-FU (as a reference drug). Mice were administered regorafenib (orally, p.o.) or 5-FU (i.p.) at doses of 30 and $8 \mathrm{mg} / \mathrm{kg} /$ day, respectively, on days $1,2,3,4,5,15,16,17,18$ and 19 , with interruption of drug administration on days 6-14 and 20-28. The treatment dose and administration route for each drug were determined based on previous studies (17-20). Each drug was dissolved in $30 \mu \mathrm{l}$ of dimethyl sulfoxide (DMSO), and then further diluted with $70 \mu \mathrm{l}$ of saline just before injecting. As a control, an equivalent volume of the vehicle was administered p.o. similar to regorafenib treatment. The mice were weighed, and their general conditions were monitored weekly. Therapeutic effects were evaluated on day 28 using 3 '- $\left[{ }^{18} \mathrm{~F}\right]$ fluoro-3'-deoxythymidine (FLT)-positron emission tomography (PET), which is a noninvasive method that is reportedly useful for evaluating the effects of chemotherapy and detecting peritoneal seeding nodules of colon cancer in clinical studies $(21,22)$. After the FLT-PET scan, the abdominal cavity was opened and then fluorescence microscopy images were acquired to confirm tumor development and detect small tumors.

FLT-PET and fluorescence microscopy imaging. FLT was synthesized as reported previously (23) and the radiochemical purity was $>99 \%$. Each mouse was intravenously injected with 3.7 MBq of FLT dissolved in $100 \mu \mathrm{l}$ of saline via the tail vein. Ten minute emission scans were performed $1 \mathrm{~h}$ after the FLT injection using a small animal PET system (Inveon, Siemens Medical Solutions, Malvern, PA, USA), while the mice were under $1.5-2 \%$ isoflurane anesthesia. Body temperature was maintained with a heat pump during the scans. The images were reconstructed using a 3D maximum a posteriori with the Inveon Acquisition Workplace software (Siemens Medical Solutions). Tracer uptake was quantified as the standard uptake value (SUV) of each voxel within the mouse abdominal 

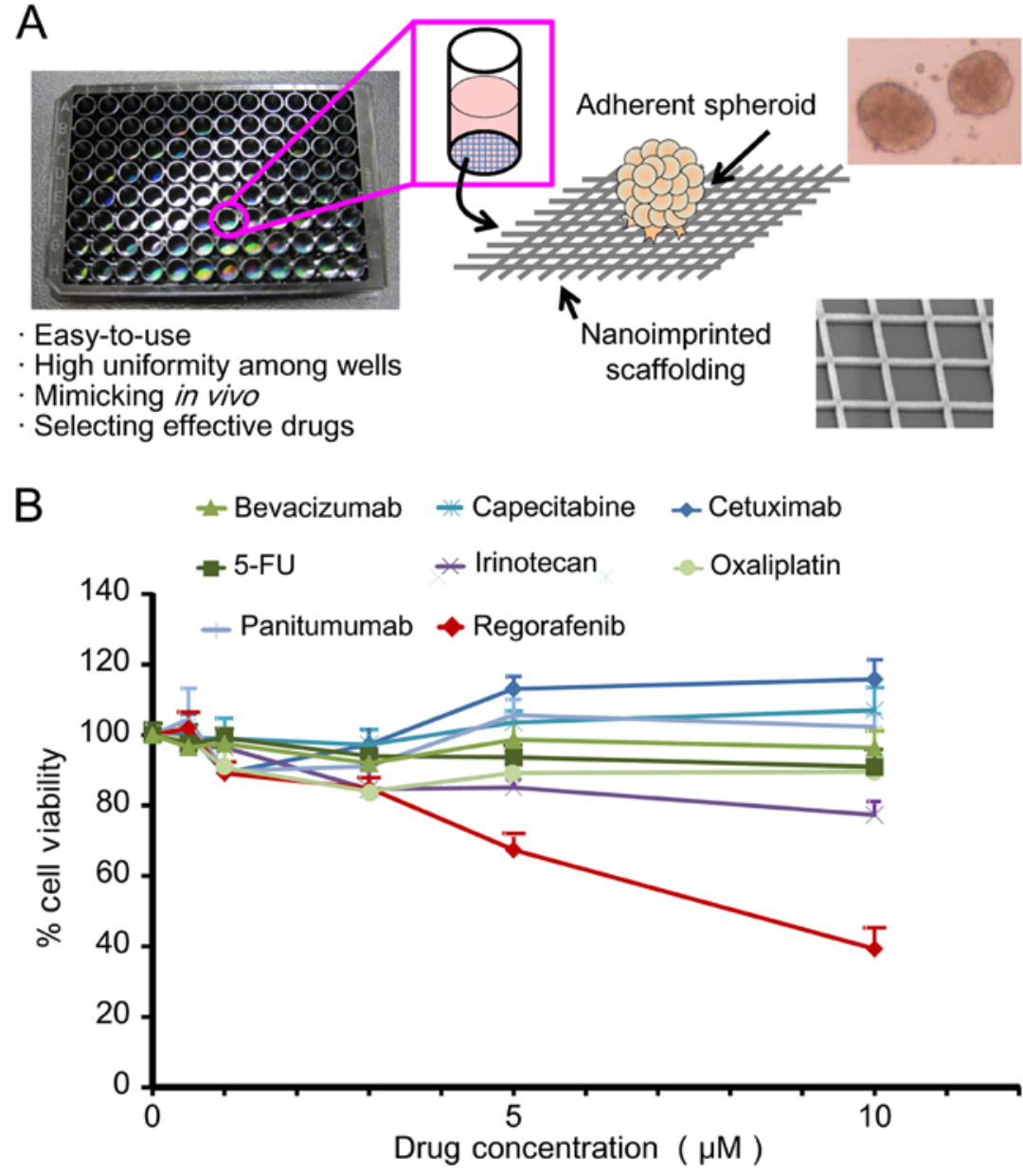

Figure 1. Drug selection using a nanoimprinting 3D culture screening system. (A) Schematic representation of screening system with nanoimprinting 3D culture (6). NCP 3D culture system consists of inorganic nanoscale scaffolding printed on transparent synthetic resinous bases using a nanoimprinting technique, which reproduces adherent cancer spheroids with characteristics resembling in vivo tumors. (B) Effect of drugs on HCT116-RFP spheroids in nanoimprinting 3D culture. Cells were treated with 8 clinically used antitumor agents for colon cancer including bevacizumab, capecitabine, cetuximab, 5-FU, irinotecan, oxaliplatin, panitumumab and regorafenib was performed. Data represent the percentage (\%) cell viability after administration of each drug at $0,0.5,1,3,5$ and $10 \mu \mathrm{M}$ relative to control $(0 \mu \mathrm{M})$. Data are mean $\pm \mathrm{SD} ; \mathrm{n}=6, \mathrm{P}<0.05$.

Table I. Anticancer drugs used in this study.

\begin{tabular}{llll}
\hline Drug & \multicolumn{1}{c}{ Target } & \multicolumn{1}{c}{ Source } & \multicolumn{1}{c}{ Location } \\
\hline Capecitabine & Nucleic acid & Sigma-Aldrich & St. Louis, MO, USA \\
5 -FU & Nucleic acid & Wako & Osaka, Japan \\
Irinotecan & Topo I & Sigma-Aldrich & St. Louis, MO, USA \\
Oxaliplatin & DNA & Tocris Bioscience & Bristol, UK \\
Cetuximab (Erbitux) & EGFR & Merck Serono & Darmstadt, Germany \\
Panitumumab (Vectibix) & EGFR & Takeda & Osaka, Japan \\
Bevacizumab (Avastin) & VEGF & Chugai & Tokyo, Japan \\
Regorafenib (Stivarga) & Multikinase & Bayer HealthCare & Leverkusen, Germany
\end{tabular}

EGFR, epidermal growth factor receptor; VEGF, vascular endothelial growth factor.

area except for the urinary bladder using the ASIPro software (CTI Molecular Imaging/Siemens) as described previously $(24,25)$. In this study, the tumor volume was calculated from the FLT-PET images as the sum of the volume of any voxel with counts greater than the fixed threshold value $(\mathrm{SUV}=1)$, which was the peak activity for the abdominal area of normal mice in the FLT-PET images.

The fluorescent images of the open mouse abdominal cavities were acquired using a fluorescent microscope (MZ16F, Leica, Wetzlar, Germany) equipped with a camera system 


\section{Control}

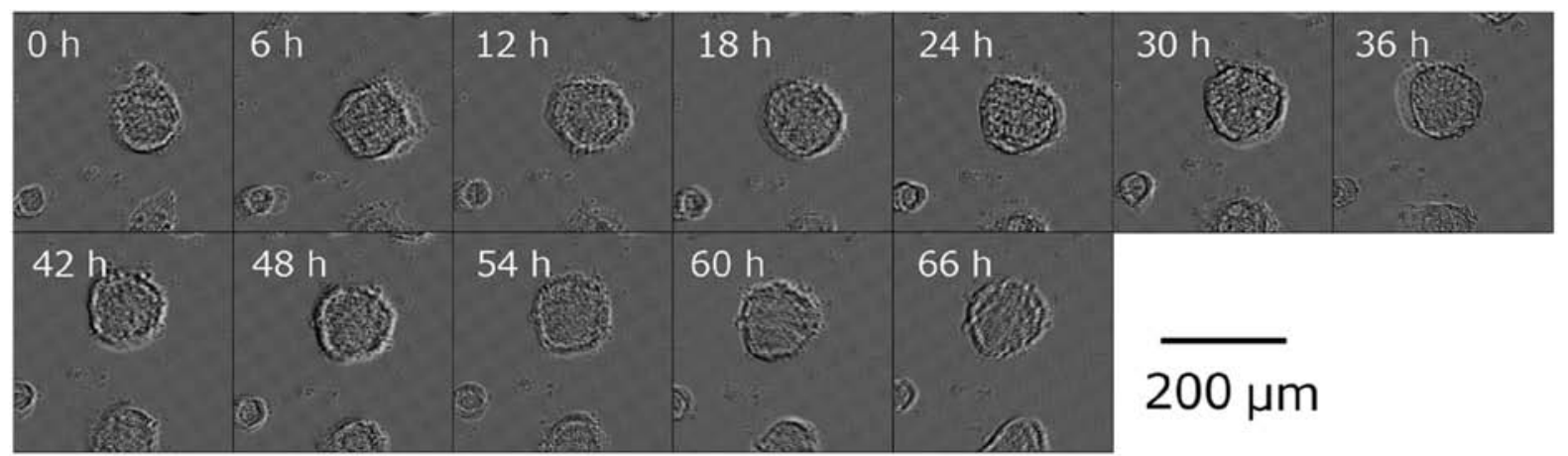

\section{5-FU}



\section{Regorafenib}

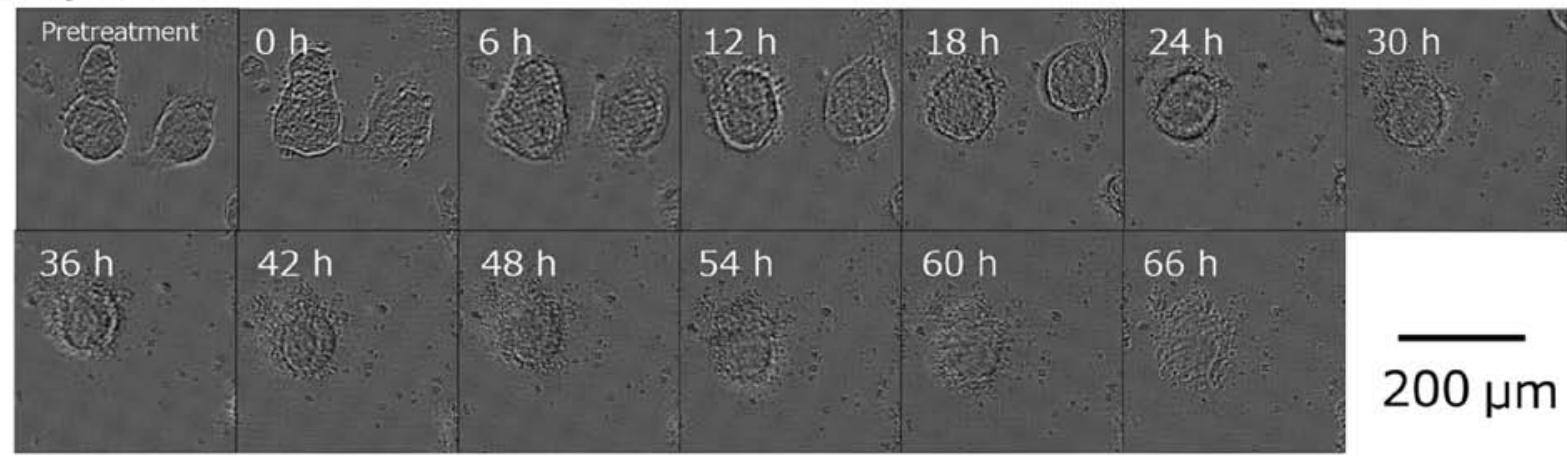

Figure 2. Effect of regorafenib on HCT116-RFP spheroids. Time-lapse microscopic bright field images acquired during incubation with $10 \mu \mathrm{M}$ regorafenib (lower panel), compared to $0 \mu \mathrm{M}$ control (upper panel) and $10 \mu \mathrm{M}$ 5-FU (reference drug, middle panel) over a 66-h period.

(DFC310FX, Leica, x0.71). Tiling images were constructed using Leica Application Suite software (Leica).

Statistical analysis. Data are expressed as the means \pm standard deviation (SD). Cell viability curves in in vitro drug selection were analyzed using two-way analysis of variance (ANOVA). Statistical significance was determined using ANOVA along with Bonferroni post hoc test for comparison of estimated tumor volume in FLT-PET study. P-values $<0.05$ were considered statistically significant.

\section{Results}

In vitro drug selection with a nanoimprinting $3 D$ culture. First, we performed in vitro drug selection using a nanoimprinting 3D culture with HCT116-RFP cells to determine the most effective candidate drug among 8 clinically used antitumor agents. Fig. 1B shows the dose-dependent effects of each drug in the HCT116-RFP spheroid viability assay. From the results, 7 drugs including bevacizumab, capecitabine, cetuximab, 5-FU, irinotecan, oxaliplatin, and panitumumab did not show the apparent decrease of the cell viability at any measured drug concentration. In contrast, regorafenib showed decrease of the cell viability by $\leq 39 \%$ at $10 \mu \mathrm{M}$ with a half-maximal inhibitory concentration $\left(\mathrm{IC}_{50}\right)$ of $8.1 \mu \mathrm{M}$. The statistical analysis on the cell viability curves showed significant inhibition in a regorafenib treatment group compared to the other treatment groups $(\mathrm{P}<0.05)$. Therefore, the in vitro drug selection using the nanoimprinting 3D culture of HCT116-RFP determined regorafenib as the most effective drug among the typical clinical antitumor agents evaluated in this study. Fig. 2 shows representative time-lapse microscopic images acquired during incubation with $10 \mu \mathrm{M}$ of regorafenib, compared to those with $0 \mu \mathrm{M}$ control and $10 \mu \mathrm{M} 5-\mathrm{FU}$ as the reference drug. The 


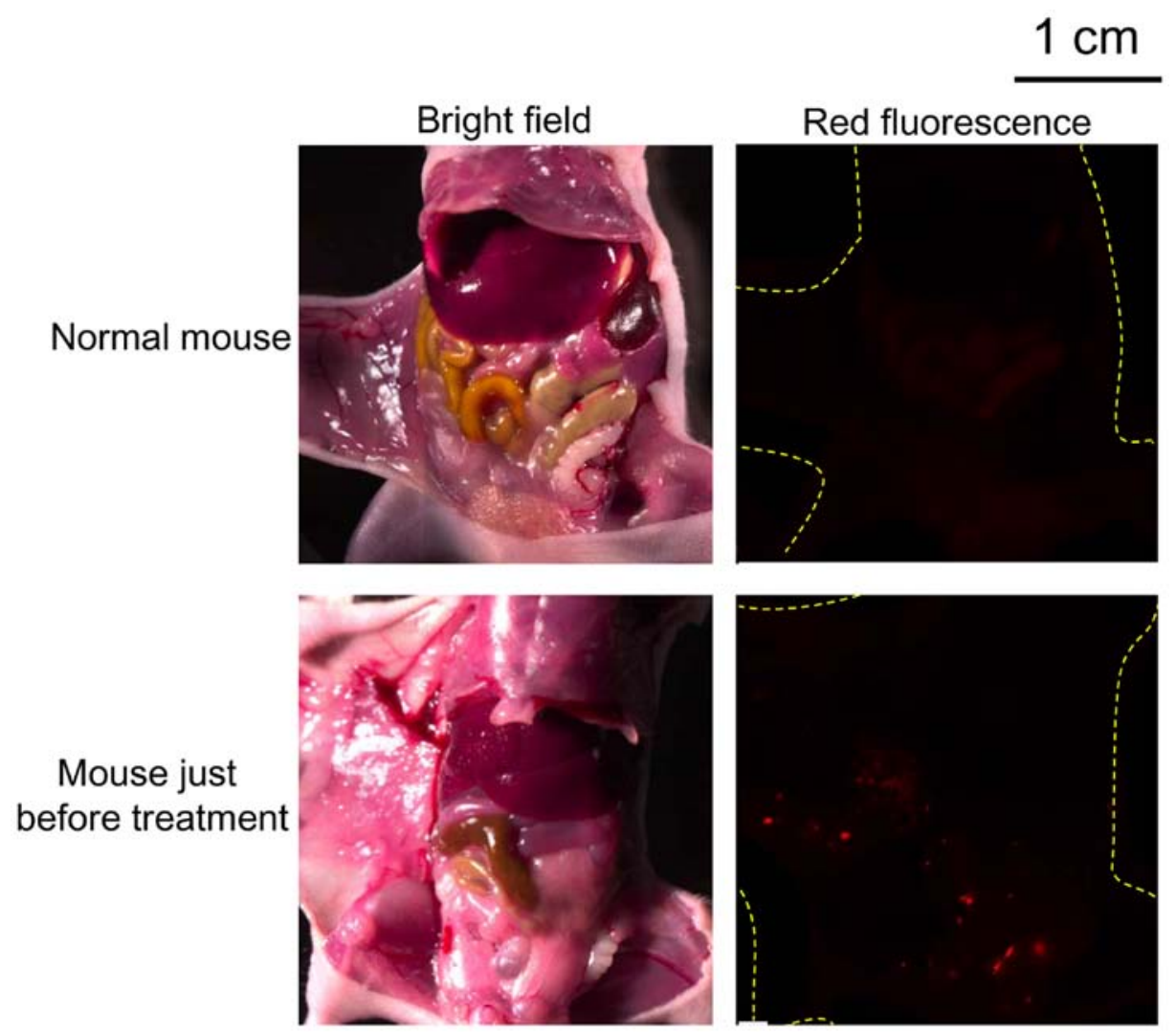

Figure 3. In vivo fluorescent imaging of mice inoculated with HCT116-RFP cells. Representative fluorescent abdominal images comparing mice intraperitoneally inoculated with and without HCT116-RFP cells (lower column and upper columns, respectively) acquired just before drug administration. Bright field and red fluorescence images (left and right rows, respectively). Images show open abdominal cavity of mice. Yellow dotted lines show outline of mice in red fluorescent images. Mice showed disseminated small HCT116-RFP tumors at time-point just before drug administration, which are indicated as bright red spots. Control mice showed low background body signals.

results demonstrate that spheroids treated with regorafenib started to collapse around $24 \mathrm{~h}$ after drug administration while the control and $10 \mu \mathrm{M}$ 5-FU-treated spheroids did not show clear morphological changes.

In vivo effects of regorafenib against disseminated tumors. Next, we tested the antitumor effects of regorafenib in a mouse model of HCT116-RFP-induced intraperitoneally disseminated tumors. Prior to the study, we confirmed that the model used in this study had developed intraperitoneally disseminated small HCT116-RFP tumors (1 mm or less) before drug administration (day 0 of the treatment), which were difficult to identify by the naked eye (Fig. 3). Using this model, we examined the effects of treatment with regorafenib and compared it to that of the untreated control and 5-FU-treated group. The evaluation was performed using the FLT-PET and fluorescence microscopy imaging.

Fig. 4A shows representative FLT-PET images of the control, 5-FU- and regorafenib-treated mice on day 28. Control and 5-FU treatment showed apparent FLT uptake in the mouse abdominal cavity while regorafenib treatment showed low uptake. Fig. 4B shows the summary of the estimated tumor volume calculated from the FLT-PET images. The regorafenib treatment group showed significantly lower estimated tumor volume than the control and 5-FU groups did $(\mathrm{P}<0.05)$. There was no significant difference between the control and 5-FU groups in estimated tumor volume from the FLT-PET analysis.
Fig. 5 shows representative fluorescence microscopy images of the opened abdominal cavities of the mice acquired after the FLT-PET scan. Highly grown and spreading small tumors are evident in the abdominal cavity of the control and 5-FU groups and less marked in the regorafenib group. Body weight loss $>10 \%$ of initial body weight and apparent changes in physical condition were not observed in the mice during regorafenib treatment.

\section{Discussion}

In this study, we found that regorafenib was the most effective drug out of 8 clinically used antitumor agents for colon cancer, using drug selection with a nanoimprinting 3D culture of colon cancer HCT116-RFP cells and demonstrated that this drug was efficacious against the in vivo disseminated small tumor model. In colon cancer treatment, surgery is usually performed as the primary treatment intervention (2). In most patients with colon cancer, the diagnosis is made at a stage when the tumor tissue can be surgically removed. However, tumor recurrence is likely to occur due to undetectable residual cancer cells in the affected patients (2). In clinical practice, patients with stage II and III colon cancers who are considered at high risk for recurrence are administered adjuvant chemotherapy $(2,4)$. 5-FU-based chemotherapy (alone or in combination with leucovorin or other drugs) is used as a standard adjuvant chemotherapy for colon cancer $(3,4)$; however, the efficacy of 

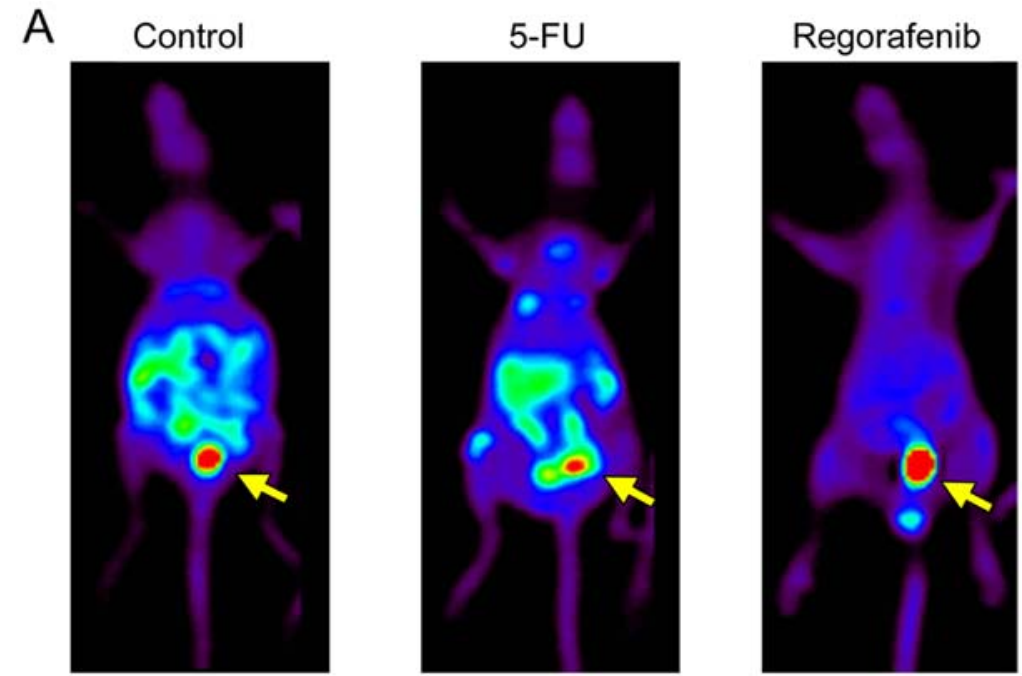

SUV mean
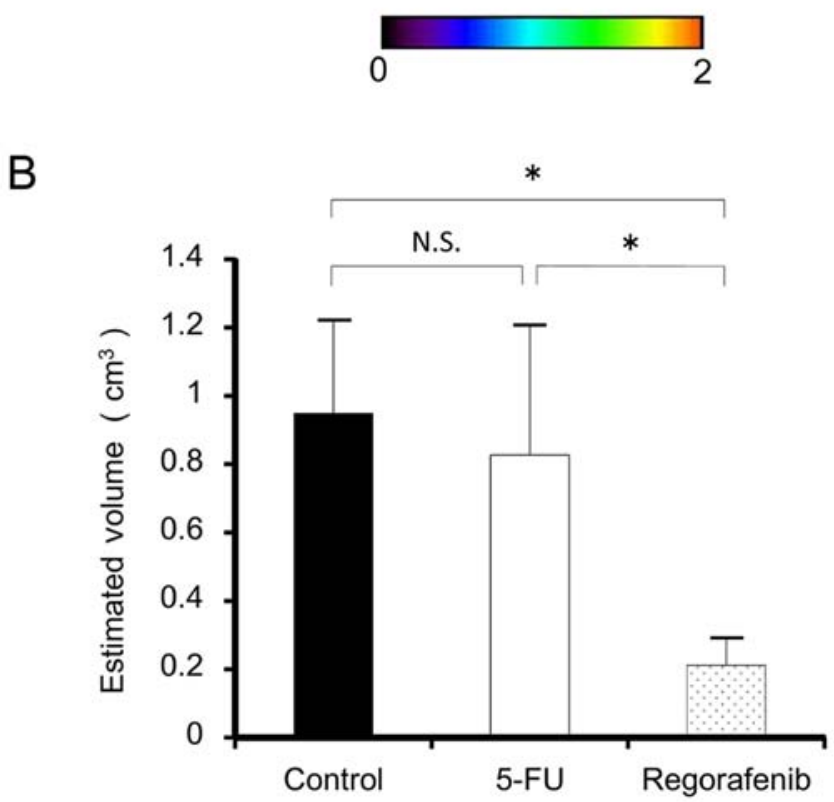

Figure 4. Evaluation of in vivo antitumor effects using FLT-PET. (A) Representative FLT-PET images of control, 5-FU- and regorafenib-treated mice (left, middle and right, respectively) on day 28. Yellow arrows indicate urinary bladder. Control and 5-FU treated groups showed elevated FLT uptake in mouse abdominal cavity (pale blue to green color). Regorafenib treatment group did not show apparent FLT uptake in mouse abdominal cavity. (B) Estimated tumor volume calculated from FLT-PET images of control, 5-FU and regorafenib groups. Data are mean $\pm \mathrm{SD}, \mathrm{n}=5 / \mathrm{group},{ }^{*} \mathrm{P}<0.05$. NS, not significant.

adjuvant chemotherapy with 5 -FU is reportedly limited and the benefit is still under contention $(3,4)$. In this study, we showed the efficacy of regorafenib against disseminated small tumors in a HCT116-RFP model. Though our findings were limited to the HCT116-RFP cell line, this study demonstrated the therapeutic potential of regorafenib as an adjuvant chemotherapy agent in colon cancer treatment.

In this study, we used a mouse model with intraperitoneally disseminated small tumors. In clinical settings, peritoneal metastasis is one of the major types of colon cancer metastasis observed at the time of primary resectioning along with liver metastasis $(26,27)$. Importantly, a higher incidence of peritoneal metastasis is reported to be observed in cases of recurrent cancer after surgery (60\%), than of liver metastasis $(50 \%)$. In addition, this phenomenon is considered to occur because of the dissemination of exfoliated primary malignancy into the free peritoneal space (26). Consequently, small undetectable tumors that are intraperitoneally disseminated before as well as after surgery are important targets in the radical cure of colon cancer. Therefore, the model used in this study is appropriate for determining the efficacy and benefits of an adjuvant chemotherapy agent for colon cancer treatment.

Regorafenib is an orally administered small molecular multikinase inhibitor that targets tumor cells, vasculature, and tumor microenvironment $(8,28)$. To date, preclinical studies with orthotopic colon cancer xenografts have revealed that regorafenib reduced tumor angiogenesis and inhibited tumor growth (20). In recent years, multinational phase III trials (CORRECT and CONCUR) have been conducted and revealed that regorafenib therapy showed survival benefits in metastatic colorectal cancer, which had progressed after standard therapies $(29,30)$. These preclinical and clinical studies showed that 

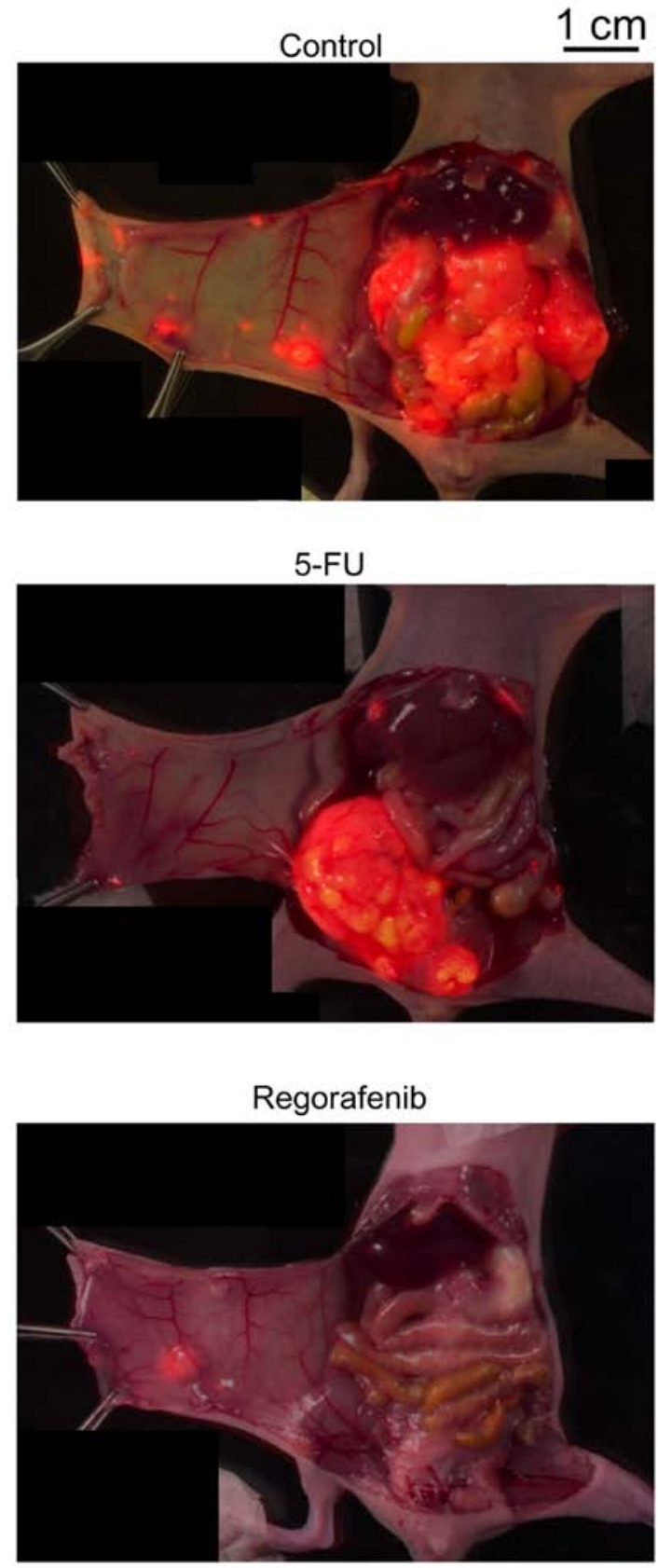

Figure 5. In vivo fluorescent imaging of mice after treatment. Representative fluorescent images of open abdominal cavities of mice after FLT-PET on day 28 of in vivo study. Control (upper), 5-FU (middle), and regorafenib (bottom) groups. Merged images of bright field and red fluorescent images are shown. Large tumor masses and spreading tiny tumors derived from HCT116-RFP cells shown as bright red signals were observed in control and 5-FU groups with less marked signals from tumors found in regorafenib group.

regorafenib is effective in primary and metastatic solid colon cancer tumors even when they had developed resistance to standard treatments. Furthermore, the present study showed that regorafenib is effective against disseminated undetectable small colon cancer. This suggests that regorafenib could be useful not only for the treatment of drug-resistant solid tumors but also for prophylactic use as an adjuvant chemotherapy agent after surgery by killing undetectable disseminated small tumors and inhibiting recurrence of colon cancer. From our in vivo study, side effects were not observed in the drug-treated mice. In clinical trials, adverse events such as hand-foot skin reaction, fatigue, diarrhea, and hypertension were observed but were manageable (31). Therefore, the side effects of regorafenib are considered minor or manageable, and this agent could be potentially beneficial for colon cancer treatment.

In this study, a novel screening system involving nanoimprinting 3D culture was used to select an effective drug against disseminated small tumors of colon cancer HCT116-RFP cells. This system consisted of nanoscale rectangular grid patterns printed on transparent synthetic resinous bases using a nanoimprinting technique and provided the optimal scaffold for cultured cancer cells to adhere to, actively migrate, and aggregate to form spheroids $(5,6)$. These characteristics of the nanoimprinting 3D culture are unique in comparison with both conventional 2D and other types of 3D cultures. In this study, we revealed that this system is useful in determining the efficacy of drugs against disseminated small tumors. This indicates that the nanoimprinting 3D culture might have a potential to facilitate the selection of effective drugs against disseminated small tumors by mimicking the characteristics of these tumors such as adhesion, migration, and proliferation as multicellular aggregates. Previous studies with HCT116 cells in $2 \mathrm{D}$ culture reported the $\mathrm{IC}_{50}$ as $4.3 \mu \mathrm{M}$ for $5-\mathrm{FU}$ (32) and 2.5-5 $\mu \mathrm{M}$ for regorafenib (33). On the other hand, in this study with the 3D culture treatment with 5-FU did not show apparent decrease in cell viability at $\leq 10 \mu \mathrm{M}$ while regorafenib showed and $\mathrm{IC}_{50}$ of $8.1 \mu \mathrm{M}$. Our in vivo study in the intraperitoneally disseminated tumor model showed that regorafenib, but not 5-FU was effective. These data indicate the nanoimprinting $3 \mathrm{D}$ culture could be a better tool for selecting effective drugs against disseminated small tumors than the 2D culture.

In conclusion, this study demonstrated the efficacy of regorafenib against disseminated small colon cancer, via drug selection using a novel screening system with a nanoimprinting 3D culture in HCT116-RFP cells. Furthermore, this drug could be a potentially useful adjuvant chemotherapy agent for colon cancer treatment and, therefore, warrants continued investigation for future clinical application.

\section{Acknowledgements}

We would like to thank Mr. Hisashi Suzuki (National Institute of Radiological Sciences, Japan) for providing the radiopharmaceuticals. This study was supported by Grants-in-Aid for Scientific Research C (grant no. 25461863) from the Japanese Society for the Promotion of Science, Japan to Y.Y.

\section{References}

1. Jemal A, Siegel R, Xu J and Ward E: Cancer statistics, 2010. CA Cancer J Clin 60: 277-300, 2010.

2. Moertel CG, Fleming TR, Macdonald JS, Haller DG, Laurie JA, Goodman PJ, Ungerleider JS, Emerson WA, Tormey DC, Glick JH, et al: Levamisole and fluorouracil for adjuvant therapy of resected colon carcinoma. N Engl J Med 322: 352-358, 1990.

3. de Vos tot Nederveen Cappel WH, Meulenbeld HJ, Kleibeuker JH, Nagengast FM, Menko FH, Griffioen G, Cats A, Morreau H, Gelderblom H, Vasen HF, et al: Survival after adjuvant 5-FU treatment for stage III colon cancer in hereditary nonpolyposis colorectal cancer. Int J Cancer 109: 468-471, 2004

4. Vaillant JC, Nordlinger B, Deuffic S, Arnaud JP, Pelissier E, Favre JP, Jaeck D, Fourtanier G, Grandjean JP, Marre P, et al: Adjuvant intraperitoneal 5-fluorouracil in high-risk colon cancer: A multicenter phase III trial. Ann Surg 231: 449-456, 2000. 
5. Yoshii Y, Waki A, Yoshida K, Kakezuka A, Kobayashi M, Namiki H, Kuroda Y, Kiyono Y, Yoshii H, Furukawa T, et al: The use of nanoimprinted scaffolds as 3D culture models to facilitate spontaneous tumor cell migration and well-regulated spheroid formation. Biomaterials 32: 6052-6058, 2011

6. Yoshii Y, Furukawa T, Waki A, Okuyama H, Inoue M, Itoh M, Zhang MR, Wakizaka H, Sogawa C, Kiyono Y, et al: Highthroughput screening with nanoimprinting 3D culture for efficient drug development by mimicking the tumor environment. Biomaterials 51: 278-289, 2015.

7. Kawada M, Inoue H, Masuda T and Ikeda D: Insulin-like growth factor I secreted from prostate stromal cells mediates tumorstromal cell interactions of prostate cancer. Cancer Res 66 : 4419-4425, 2006.

8. Wilhelm SM, Dumas J, Adnane L, Lynch M, Carter CA, Schütz G, Thierauch KH and Zopf D: Regorafenib (BAY 73-4506): A new oral multikinase inhibitor of angiogenic, stromal and oncogenic receptor tyrosine kinases with potent preclinical antitumor activity. Int J Cancer 129: 245-255, 2011.

9. Schmieder R, Hoffmann J, Becker M, Bhargava A, Müller T, Kahmann N, Ellinghaus P, Adams R, Rosenthal A, Thierauch KH, et al: Regorafenib (BAY 73-4506): Antitumor and antimetastatic activities in preclinical models of colorectal cancer. Int J Cancer 135: 1487-1496, 2014

10. Kehoe SM, Ma C, Rosales N, Rao T, Dupont J and Spriggs DR: Effect of combination inhibition of vascular endothelial growth factor (VEGF) and epidermal growth factor receptor (EGF-R) on ovarian cancer cell lines. J Clin Oncol 24 (Suppl 13112): 2006.

11. Kunnumakkara AB, Diagaradjane P, Anand P, Harikumar KB, Deorukhkar A, Gelovani J, Guha S, Krishnan S and Aggarwal BB: Curcumin sensitizes human colorectal cancer to capecitabine by modulation of cyclin D1, COX-2, MMP-9, VEGF and CXCR4 expression in an orthotopic mouse model. Int J Cancer 125: 2187-2197, 2009

12. Yamamoto Y, Fukuda K, Fuchimoto Y, Matsuzaki Y, Saikawa Y, Kitagawa Y, Morikawa Y and Kuroda T: Cetuximab promotes anticancer drug toxicity in rhabdomyosarcomas with EGFR amplification in vitro. Oncol Rep 30: 1081-1086, 2013.

13. Jiang P, Mukthavaram R, Chao Y, Bharati IS, Fogal V, Pastorino S, Cong X, Nomura N, Gallagher M, Abbasi T, et al: Novel antiglioblastoma agents and therapeutic combinations identified from a collection of FDA approved drugs. J Transl Med 12: 13, 2014.

14. Dahan L, Sadok A, Formento JL, Seitz JF and Kovacic H: Modulation of cellular redox state underlies antagonism between oxaliplatin and cetuximab in human colorectal cancer cell lines. Br J Pharmacol 158: 610-620, 2009.

15. Freeman DJ, Bush T, Ogbagabriel S, Belmontes B, Juan T, Plewa C, Van G, Johnson C and Radinsky R: Activity of panitumumab alone or with chemotherapy in non-small cell lung carcinoma cell lines expressing mutant epidermal growth factor receptor. Mol Cancer Ther 8: 1536-1546, 2009.

16. Cordel S, Heymann MF, Boisteau O, Oliver L, Le Pendu J, Grégoire $\mathrm{M}$ and Meflah K: 5-Fluorouracil-resistant colonic tumors are highly responsive to sodium butyrate/interleukin-2 bitherapy in rats. Int J Cancer 73: 924-928, 1997.

17. Van Looy T, Gebreyohannes YK, Wozniak A, Cornillie J, Wellens J, Li H, Vanleeuw U, Floris G, Debiec-Rychter M, Sciot R, et al: Characterization and assessment of the sensitivity and resistance of a newly established human gastrointestinal stromal tumour xenograft model to treatment with tyrosine kinase inhibitors. Clin Sarcoma Res 4: 10, 2014

18. Gold GL, Hall TC, Shnider BJ, Selawry O, Colsky J, Owens AH Jr, Dederick MM, Holland JF, Brindley CO and Jones R: A clinical study of 5-fluorouracil. Cancer Res 19: 935-939, 1959.

19. Miyake M, Anai S, Fujimoto K, Ohnishi S, Kuwada M, Nakai Y, Inoue $\mathrm{T}$, Tomioka $\mathrm{A}$, Tanaka $\mathrm{N}$ and Hirao $\mathrm{Y}$ : 5-fluorouracil enhances the antitumor effect of sorafenib and sunitinib in a xenograft model of human renal cell carcinoma. Oncol Lett 3 . 1195-1202, 2012.
20. Abou-Elkacem L, Arns S, Brix G, Gremse F, Zopf D, Kiessling F and Lederle W: Regorafenib inhibits growth, angiogenesis, and metastasis in a highly aggressive, orthotopic colon cancer model. Mol Cancer Ther 12: 1322-1331, 2013.

21. Wieder HA, Geinitz H, Rosenberg R, Lordick F, Becker K, Stahl A, Rummeny E, Siewert JR, Schwaiger M and Stollfuss J: PET imaging with $\left[{ }^{18} \mathrm{~F}\right] 3$ '-deoxy-3'-fluorothymidine for prediction of response to neoadjuvant treatment in patients with rectal cancer. Eur J Nucl Med Mol Imaging 34: 878-883, 2007.

22. Hong YS, Kim HO, Kim KP, Lee JL, Kim HJ, Lee SJ, Lee SJ, Oh SJ, Kim JS, Ryu JS, et al: 3'-Deoxy-3'- ${ }^{18} \mathrm{~F}$-fluorothymidine PET for the early prediction of response to leucovorin, 5-fluorouracil, and oxaliplatin therapy in patients with metastatic colorectal cancer. J Nucl Med 54: 1209-1216, 2013.

23. Wodarski CEJ, Weber K, Henze M, Haberkorn U and Eisenhut M: Synthesis of 3'-deoxy-3'-[ ${ }^{18}$ F]fluoro-thymidine with 2,3'-anhydro-5'-O-(4,4'-dimethoxytrityl)-thymidine. J Labelled Comp Radiopharm 43: 1211-1218, 2000.

24. Tsuji AB, Kato K, Sugyo A, Okada M, Sudo H, Yoshida C, Wakizaka H, Zhang MR and Saga T: Comparison of 2-amino[3-11 C]isobutyric acid and 2-deoxy-2- $\left[{ }^{18} \mathrm{~F}\right]$ fluoro-D-glucose in nude mice with xenografted tumors and acute inflammation. Nucl Med Commun 33: 1058-1064, 2012.

25. Tsuji AB, Morita M, Li XK, Sogawa C, Sudo H, Sugyo A, Fujino M, Sugioka A, Koizumi M and Saga T: ${ }^{18}$ F-FDG PET for semiquantitative evaluation of acute allograft rejection and immunosuppressive therapy efficacy in rat models of liver transplantation. J Nucl Med 50: 827-830, 2009.

26. Sugarbaker PH: Colorectal cancer: Prevention and management of metastatic disease. BioMed Res Int 2014: 782890, 2014

27. Thomassen I, van Gestel YR, Lemmens VE and de Hingh IH: Incidence, prognosis, and treatment options for patients with synchronous peritoneal carcinomatosis and liver metastases from colorectal origin. Dis Colon Rectum 56: 1373-1380, 2013.

28. Brodowicz T, Liegl-Atzwager B, Tresch E, Taieb S, Kramar A, Gruenwald V, Vanseymortier M, Clisant S, Blay JY, Le Cesne A, et al: Study protocol of REGOSARC trial: activity and safety of regorafenib in advanced soft tissue sarcoma: a multinational, randomized, placebo-controlled, phase II trial. BMC Cancer 15: $127,2015$.

29. Grothey A, Van Cutsem E, Sobrero A, Siena S, Falcone A, Ychou M, Humblet Y, Bouché O, Mineur L, Barone C, et al; CORRECT Study Group: Regorafenib monotherapy for previously treated metastatic colorectal cancer (CORRECT): An international, multicentre, randomised, placebo-controlled, phase 3 trial. Lancet 381: 303-312, 2013.

30. Li J, Qin S, Xu R, Yau TC, Ma B, Pan H, Xu J, Bai Y, Chi Y, Wang L, et al; CONCUR Investigators: Regorafenib plus best supportive care versus placebo plus best supportive care in Asian patients with previously treated metastatic colorectal cancer (CONCUR): A randomised, double-blind, placebo-controlled, phase 3 trial. Lancet Oncol 16: 619-629, 2015.

31. Jitawatanarat $P$ and Wee W: Update on antiangiogenic therapy in colorectal cancer: Aflibercept and regorafenib. J Gastrointest Oncol 4: 231-238, 2013

32. Boyer J, McLean EG, Aroori S, Wilson P, McCulla A, Carey PD, Longley DB and Johnston PG: Characterization of p53 wild-type and null isogenic colorectal cancer cell lines resistant to 5-fluorouracil, oxaliplatin, and irinotecan. Clin Cancer Res 10: 2158-2167, 2004

33. Fan LC, Teng HW, Shiau CW, Lin H, Hung MH, Chen YL, Huang JW, Tai WT, Yu HC and Chen KF: SHP-1 is a target of regorafenib in colorectal cancer. Oncotarget 5: 6243-6251, 2014. 